\section{Geographic Distribution of Raccoon Roundworm, Baylisascaris procyonis, Germany and Luxembourg}

Mike Heddergott, Peter Steinbach, Sabine Schwarz, Helena E. Anheyer-Behmenburg, Astrid Sutor, Annette Schliephake, Diana Jeschke, Michael Striese, Franz Müller, Elisabeth MeyerKayser, Michael Stubbe, Natalia Osten-Sacken, Susann Krüger, Wolfgang Gaede, Martin Runge, Lothar Hoffmann, Hermann Ansorge,

Franz J. Conraths, Alain C. Frantz

Author affiliations: Musée National d'Histoire Naturelle, Luxembourg, Luxembourg (M. Heddergott, P. Steinbach, A.C. Frantz); Georg-August University, Göttingen, Germany (P. Steinbach); Friedrich-Loeffler-Institut, Greifswald-Insel Riems, Germany (S. Schwarz, A. Sutor, F.J. Conraths); Lower Saxony State Office for Consumer Protection and Food Safety, Hannover, Germany (H.E. Anheyer-Behmenburg, M. Runge); State Office for Consumer Protection Saxony-Anhalt, Stendal, Germany (A. Schliephake, W. Gaede); Senckenberg Museum of Natural History Görlitz, Görlitz, Germany (D. Jeschke, M. Striese, H. Ansorge); Justus-Liebig-University Giessen, Giessen, Germany
(F. Müller); Thuringia Office for Consumer Protection, Bad Langensalza, Germany (E. Meyer-Kayser, L. Hoffmann); Martin-Luther University Halle-Wittenberg, Halle/Saale, Germany (M. Stubbe); Nicolaus Copernicus University, Toruń, Poland (N. Osten-Sacken); Fondation Faune-Flore, Luxembourg (N. Osten-Sacken); German Hunting Association, Berlin, Germany (A. Sutor, S. Krüger); International Institute Zittau, Technische Universität Dresden, Zittau, Germany (H. Ansorge)

DOI: https://doi.org/10.3201/eid2604.191670

Infestation with Baylisascaris procyonis, a gastrointestinal nematode of the raccoon, can cause fatal disease in humans. We found that the parasite is widespread in central Germany and can pose a public health risk. The spread of $B$. procyonis roundworms into nematode-free raccoon populations needs to be monitored.

$\mathrm{T}$ he raccoon roundworm (Baylisascaris procyonis) is a gastrointestinal parasitic nematode of the raccoon (Procyon lotor). It is common in its native range in North America, where its prevalence in raccoons can reach $82 \%$ (1). Through their feces, infested raccoons can shed millions of $B$. procyonis eggs, which may remain infective in the environment for years (2). Paratenic hosts can acquire the parasite when ingesting nematode eggs from raccoon latrines (3).

\title{
etymologia
}

\section{Trombiculiasis [trom-bik"u-li'ə-sis]}

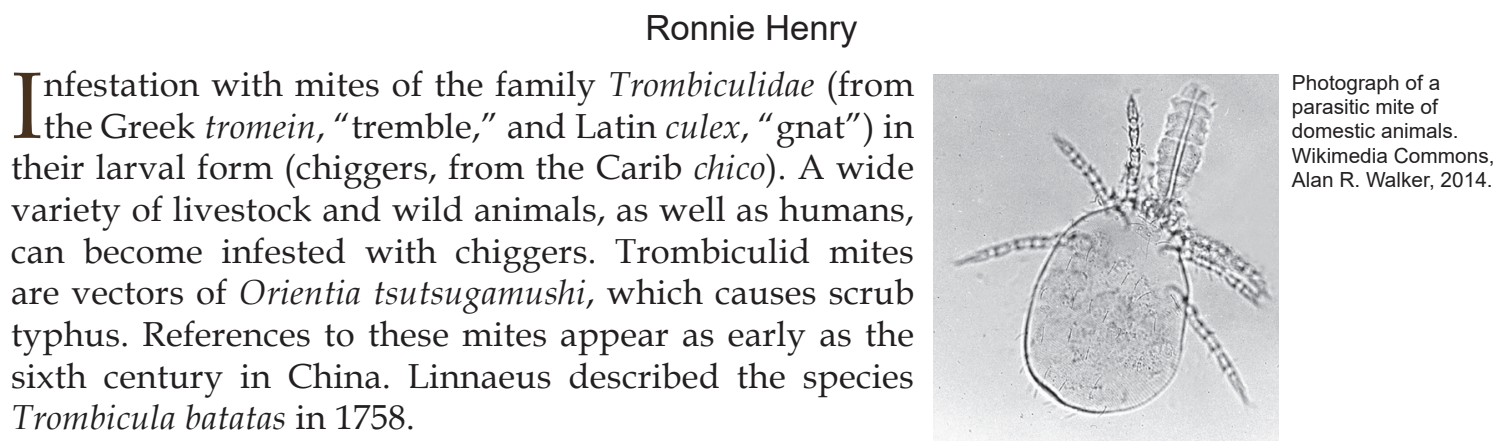

\section{Sources}

1. Bowman DD, Hendrix CM, Lindsay DS, Barr SC. Feline clinical parasitology. Ames (IA): Iowa State University Press; 2002.
2. Scarborough J. Medical and biological terminologies: classical origins. Norman (OK): University of Oklahoma Press; 1992.

Address for correspondence: Ronnie Henry, Centers for Disease Control and Prevention, 1600 Clifton Rd NE, Mailstop V18-2, Atlanta, GA 30329-4027, USA; email: boq3@cdc.gov 